\title{
Senile disciform macular degeneration: features indicating suitability for photocoagulation
}

\author{
R. H. B. GREY, A. C. BIRD, AND I. H. CHISHOLM \\ From the department of Clinical Ophthalmology, Institute of Ophthalmology and Moorfields Eye Hospital, \\ London
}

SUMMARY During a 1-year period 398 new patients were seen with disciform macular degeneration (530 eyes). The lesions were studied retrospectively, and those in which the neovascular tissue did not underlie the fovea, and therefore were treatable, were identified. The following conclusions were drawn: (1) Treatment is possible in most patients with good acuity and few with poor acuity. (2) Treatment is possible in a large proportion of patients with a short history and few with a long history of visual loss. (3) As many as $50 \%$ of all patients with senile disciform macular degeneration may be amenable to treatment if seen early enough in the course of their disease. (4) Over one-third of eyes with lesions that are untreated have a visual acuity of $6 / 60$ or better 3 years after the onset of symptoms. (5) If a controlled trial proves that treatment is beneficial, these results emphasise the need for rapid referral and show that these patients will generate a large additional clinical load.

The early reports of photocoagulation in senile disciform macular degeneration showed poor visual results after treatment (Zweng et al., 1968; Schneider, 1970; l'Esperance, 1971). With a better understanding of the disease process (Gass, 1967; Teeters and Bird, 1973a,b) a more rational approach to photocoagulation has evolved. This, coupled with the introduction of argon laser photocoagulation and its adaptation to the slit lamp ('Esperance, 1968; Little et al., 1970) has given rise to better visual results (Schatz and Patz, 1973; Gass, 1973; Bird, 1974; Bonnet, 1975; Hirose, 1976; Sand, 1977).

The most recent experiences suggest that photocoagulation for disciform macular disease may be justifiable and certainly indicate the need for a randomised clinical trial to test its true value.

A variety of factors have been identified during feasibility studies which suggest that the value of photocoagulation may be limited. Photocoagulation does nothing to alter the basic disease process, so that recurrences can be expected (Bird, 1975). Secondly, it has become evident that only a minority of disciform lesions are amenable to therapy when first seen because the neovascular tissue underlies the fovea in most patients.

This retrospective study was undertaken to determine the percentage of disciform lesions

Address for reprints: Mr R. H. B. Grey, FRCS, Moorfields Eye Hospital, City Road, London ECIV 2PD referred to the Retinal Diagnostic Department at this hospital which were amenable to photocoagulation and to identify those clinical attributes which may guide the clinician as to the likelihood of the lesion being suitable for photocoagulation. This information may give some indication about the management of disciform macular disease, and what the size of the clinical load might be if clinical trial shows this form of treatment to be justifiable.

\section{Materials and methods}

Fluorescein angiograms of all new non-diabetic patients referred to the Retinal Diagnostic Department of Moorfields Eye Hospital were examined retrospectively for the 12-month period from 1 December 1976 to 5 December 1977. Those angiograms which were found to have neovascular disciform lesions or pigment epithelial detachments were further subdivided according to the supposed aetiology of the disease.

Senile disciform degeneration was diagnosed in those patients over 50 years of age with drusen and pigment epithelial changes in addition to subretinal neovascularisation and in whom there was no evidence of other cause for their disease. The visual acuity and the duration of symptoms at the time of angiography were recorded for each affected eye.

Treatment by argon laser photocoagulation was considered possible in those eyes in which the sub- 
retinal neovascular membrane could be clearly defined and in which there was a discernible gap between the edge of the neovascular tissue and the foveola (about $100 \mu \mathrm{m}$ ). The possibility of argon laser treatment at the time of angiography was related to the visual acuity and the duration of symptoms in affected eyes.

Patients in the older age group with pigment epithelial detachments associated with drusen were also identified, since these may precede the onset of vascular disciform lesions (Gass, 1967; Teeters and Bird, 1973b). All such lesions were considered potentially treatable.

All those patients who had had disciform degeneration for 3 years or more before angiography were analysed in order to identify the prognosis for visual acuity in untreated eyes.

\section{Results}

Two thousand non-diabetic patients were seen during the 12-month period of the study, and 530 eyes (398 patients) had subretinal neovascularisation (Table 1); $20 \%$ of all senile disciform lesions were potentially treatable at the time they were first seen; this compares with $32 \%$ in the younger group.

Table 2 shows the visual acuities of the patients with senile subretinal neovascular lesions at the time of fluorescein angiography. Argon laser treatment was theoretically possible in $90 \%$ of cases with an acuity of $6 / 12$ or better. Conversely, the lesions were found to be untreatable in over $98 \%$ of cases with vision as low as $6 / 60$, and no treatable cases were found once the vision had deteriorated to below $6 / 60$. Fig. 1 shows the reduction of the number of treatable disciform lesions with the drop in visual acuity. Well over half the eyes ( 255 of $443 ; 58 \%$ ) when first seen fell into the group with an acuity of $6 / 60$ or worse, and therefore there was little chance of their being suitable for treatment.

Table 1 Number of eyes with treatable and untreatable subretinal neovascularisation and pigment epithelial detachments

\begin{tabular}{lcclc}
\hline & Treatable & Untreatable & $\begin{array}{l}\text { Pigment } \\
\text { epithelial } \\
\text { detachment }\end{array}$ & Total \\
\hline Senile & 90 & 355 & 63 & 508 \\
POHS* & 9 & 11 & 0 & 20 \\
Myopia & 4 & 24 & 0 & 28 \\
Others & 14 & 23 & 3 & 40 \\
\hline Total & 117 & 413 & 66 & 596 \\
\hline
\end{tabular}

* Presumed ocular histoplasmosis syndrome.
The length of time the patients were symptomatic before fluorescein angiography was found to be related to the number of treatable senile disciform lesions (Table 3, Fig. 2); $83 \%$ of lesions were treatable when symptoms had been present for 2 weeks or less. This percentage fell to $43 \%$ at 1 month and to $30 \%$ at 4 months. When symptoms had been

Table 2 Number of treatable senile neovascular disciform lesions related to visual acuity at angiography (1 treatable and 1 untreatable eye had no acuity recorded)

\begin{tabular}{cccccc}
\hline \multirow{2}{*}{ Acuity } & \multicolumn{2}{l}{ Treatable } & \multicolumn{2}{l}{ Untreatable } & \multirow{2}{*}{ Total } \\
\cline { 2 - 5 } & No. & $(\%)$ & No. & $(\%)$ & \\
\hline $6 / 6$ & 3 & $(100)$ & 0 & $(0)$ & 3 \\
$6 / 9$ & 16 & $(84)$ & 3 & $(16)$ & 19 \\
$6 / 12$ & 15 & $(94)$ & 1 & $(6)$ & 16 \\
$6 / 18$ & 32 & $(48)$ & 34 & $(52)$ & 66 \\
$6 / 24$ & 11 & $(31)$ & 24 & $(69)$ & 35 \\
$6 / 36$ & 9 & $(20)$ & 37 & $(80)$ & 46 \\
$6 / 60$ & 3 & $(4)$ & 67 & $(96)$ & 70 \\
$<6 / 60$ & 0 & $(0)$ & 188 & $(100)$ & 188 \\
\hline Total & 89 & $(20)$ & 354 & $(80)$ & 443 \\
\hline
\end{tabular}

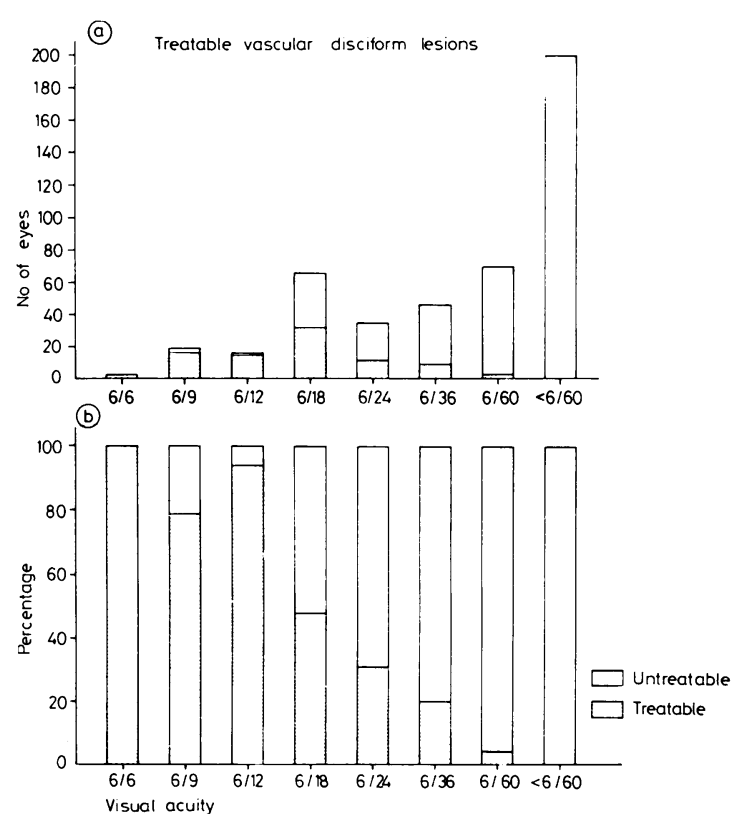

Fig. 1 (a) Number of treatable and untreatable patients related to visual acuity at angiography. (b) Percentage of treatable patients of each acuity 
Table 3 Number of treatable senile neovascular disciform lesions related to duration of symptoms ( 3 treatable and 18 untreatable eyes hid no exact duration recorded)

\begin{tabular}{llllll}
\hline \multirow{2}{*}{$\begin{array}{l}\text { Duration of } \\
\text { symptoms }\end{array}$} & \multicolumn{2}{l}{ Treatable } & \multicolumn{2}{l}{ Untreatable } & Total \\
\cline { 2 - 5 } No. & $(\%)$ & No. & $(\%)$ & \\
\hline 0-2 weeks & 10 & $(83)$ & 2 & $(17)$ & 12 \\
3-4 weeks & 15 & $(43)$ & 20 & $(57)$ & 35 \\
5-8 weeks & 11 & $(35)$ & 20 & $(65)$ & 31 \\
9-12 weeks & 21 & $(47)$ & 24 & $(53)$ & 45 \\
3-4 months & 6 & $(30)$ & 14 & $(70)$ & 20 \\
5-6 months & 5 & $(10)$ & 47 & $(90)$ & 52 \\
7-8 months & 2 & $(12)$ & 14 & $(88)$ & 16 \\
8-12 months & 7 & $(11)$ & 57 & $(89)$ & 64 \\
Over 12 months & 1 & $(1)$ & 125 & $(99)$ & 126 \\
Asymptomatic & 9 & $(69)$ & 4 & $(31)$ & 13 \\
\hline Total & 87 & $(21)$ & 327 & $(79)$ & 414 \\
\hline
\end{tabular}
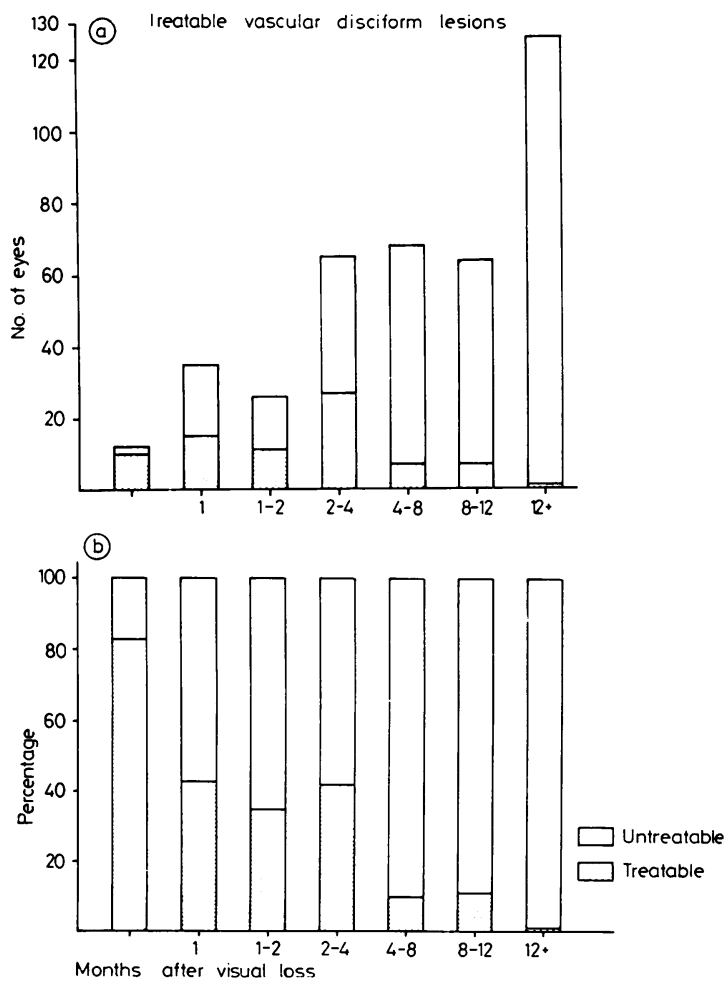

Fig. 2 (a) Number of treatable and untreatable patients related to duration of symptoms. (b) Percentage of treatable patients related to duration of symptoms present for over 1 year, only $1 \%$ of the eyes had treatable lesions. The group of eyes which could have been predicted as being untreatable because symptoms started more than 4 months previously were a majority of those seen $(243$ of $414 ; 59 \%)$.

Thirteen patients with senile disciform degeneration in 1 eye had asymptomatic lesions in the fellow eye; $69 \%$ of these asymptomatic lesions were treatable.

Pigment epithelial detachments were found in 63 eyes in the group of patients with senile degeneration. These included both subfoveal and parafoveal detachments, and all were considered to be theoretically treatable except those in which the lesion was so small that photocoagulation would have involved the fovea.

Disciform lesions which had been present for 3 years or more had a better prognosis in the younger patients than in the elderly. Ten out of 15 younger patients $(67 \%)$ had a final acuity of $6 / 60$ or better and $5(33 \%)$ had $6 / 24$ or better. In the older group 24 out of $76(32 \%)$ had $6 / 60$ or better.

\section{Discussion}

The proportion of treatable lesions obtained in this series is not necessarily representative of disciform degeneration in the general ophthalmic outpatient population of this hospital owing to prior selection for fluorescein angiography; patients with longstanding disciform changes are less frequently investigated. Thus, in the eyes with senile neovascular changes the treatable number obtained here of $20 \%$ (30\% including pigment epithelial detachments) may be too high in relation to the total number of patients with senile disciform disease presenting to the hospital clinics. On the other hand some eyes included in the study did not afford the primary reason for the patients' referral to us, since it was the fellow eye of one with recent visual loss. However, analysis of those patients who were considered treatable may offer useful guides to the clinician when trying to assess the likelihood of treatment being possible.

The chance of a disciform lesion being treatable may be predicted from the visual acuity alone. In this study an affected eye with an acuity of $6 / 24$ or better had at least a 1-in-3 chance of having a treatable lesion, but once the acuity had fallen to $6 / 60$ or worse the chances were remote.

More important was the correlation between the proportion of treatable lesions in patients and the length of history of visual loss. $53 \%$ seen within 1 month were treatable, whereas the figure was only $15 \%$ when the symptoms had lasted over 4 months and $1 \%$ when over 1 year. This is not surprising, 
since progression is to be expected. Subretinal neovascular tissue, which originates eccentrically, has a tendency to expand, with a predilection for growth towards the fovea (Teeters and Bird, 1973b). This explains not only the loss of visual acuity but also the progression to being untreatable with the passage of time. This predictability of progression is important. It might be argued that a lesion in which the neovascular tissue does not underlie the fovea after 4 months is unlikely to progress further and may not need therapy. The fact that only 1 patient with a long history had a treatable lesion would suggest that this is not valid. However, with longstanding untreated lesions the ultimate visual outcome would argue against photocoagulation of subfoveal neovascular tissue. That $32 \%$ of older patients and $67 \%$ of younger patients retained or regained an acuity of $6 / 60$ or better renders photocoagulation of the fovea difficult to justify.

It is relevant that a relatively large proportion of lesions were suitable for photocoagulation when seen early in their disease. There is no reason to believe that those seen early were different from those seen late, and it is likely that a much larger proportion of the latter group would have been treatable had they been seen earlier. The proportion of treatable lesions is encouraging, and is much higher than we expected. These figures imply that if photocoagulation proves to be an effective form of therapy it could be available to a large number of patients if systems of referral and assessment are speeded up.

The present justifiable demand that new therapeutic agents be assessed by controlled trial before they become accepted into routine medical practice gives rise to new problems in organising the delivery of health care. This is particularly important if the new form of treatment requires expensive equipment and additional staff. The treatment remains experimental before the trial is completed and yet if it is shown to be effective by trial it becomes justifiable and should be universally available. The former practice, which allowed the gradual evolution of new therapy over a period of decades, has been replaced by a situation where a rapid solution is required. The management of diabetic retinopathy recently proved (Diabetic Research Group, 1976; Cheng, 1976) illustrates the difficulties generated by the advent of clinical trials. Therefore, before a trial is completed, information is required on the natural history and population incidence of the disease in order to plan in advance the provision of the new therapy.

Clearly, if controlled trials show that patients with treatable disciform lesions derive benefit from photocoagulation, systems of referral and treatment ought to take account of our observations if they are representative of the disease. A large proportion of lesions are treatable if seen early in the course of the disease, but extension of the subretinal neovascular tissue beneath the foveola will occur in nearly all cases, and photocoagulation of such advanced lesions is unlikely to be justifiable. Once disciform macular disease is identified the patient should be investigated urgently. Delays may occur between the request from the patient to be seen and the first examination by the ophthalmologist, in providing and assessing the fluorescein angiogram, and in initiating therapy. Inordinate delay at any stage may render a treatable condition untreatable. The late arrival of a large proportion of the patients at our clinic attests to the presence of such delays in the system of referral. Education of the patients (and in Britain of general practitioners) would reduce the interval between the onset of symptoms and the first request by the patient for medical help. This could certainly be achieved in the patients who have sought ophthalmic help because 1 eye had already been affected, for in them there is a risk of the second eye being affected of $12 \%$ per year (Gregor et al., 1977).

If controlled trial shows photocoagulation to be beneficial the clinical load which may be generated by disciform degeneration and its treatment will depend on its prevalence and the proportion of lesions likely to be treatable. There is no doubt that disciform macular degeneration is common. It has been estimated to account for 35\% (Sorsby, 1966) of the blind population in Caucasian societies, and the incidence of visible macular changes in the elderly of such societies is estimated as being $30 \%$ (Kornschweig et al., 1957). The figures in this study suggest that photocoagulation may be justifiable in a large proportion of lesions, so that the additional clinical load could be great. Prompt referral and ready availability of treatment facilities will be ncessary to take advantage of this form of therapy.

We thank Mr K. Sehmi for drawing the graphs and Miss Jayne Fisher for typing the manuscript.

References

Bird, A. C. (1974). Treatment of senile disciform macular degeneration by photocoagulation. British Journal of Ophthalmology, 58, 367-376.

Bird, A. C. (1975). The macular disciform response and laser treatment. Documenta Ophthalmologica Proceedings Series: New Developments in Ophthalmology, 7, 205-212.

Bonnet, M. (1975). Photocoagulation des dégénérescences maculaires séniles. Archives d'Ophtalmologie, 35, 311-326.

Cheng, H. (1976). Response of proliferative diabetic retinopathy to xenon arc photocoagulation: a multi-centre randomised controlled trial. Transactions of the Ophthalmological Societies of the United Kingdom, 96, 224-227. 
Diabetic Research Group (1976). Preliminary report on effects of photocoagulation therapy. American Journal of Ophthalmology, 81, 383-396.

Gass, J. D. M. (1967). Pathogenesis of disciform detachment of the neuroepithelium: Part III. Senile disciform macular degeneration. American Journal of Ophthalmology, 63, 617-645.

Gass, J. D. M. (1973). Drusen and disciform macular detachment and degeneration. Archives of Ophthalmology, 90, 206-217.

Gregor, Z., Bird, A. C., and Chisholm, I. H. (1977). Senile disciform macular degeneration in the second eye. British Journal of Ophthalmology, 61, 141-147.

Hirose, T. (1976). Argon laser coagulation in subretinal neovascular membranes near the posterior pole. Treatment for senile disciform macular degeneration. Japanese Journal of Clinical Ophthalmology, 30, 191-198.

Kornschweig, A. L., Feldstein, M., and Schneider, J. (1957). The eye in old age. American Journal of Ophthalmology, 44, 29-37.

l'Esperance, F. A. (1968). An ophthalmic argon laser photocoagulation system: design, construction and laboratory investigation. Transactions of the American Ophthalmological Society, 66, 827-904.

l'Esperance, F. A. (1971). Argon and ruby laser photocoagulation of disciform macular degeneration. Transac- tions of the American Academy of Ophthalmology and Otolaryngology, 75, 609-625.

Little, H. L., Zweng, H. C., and Peabody, R. R. (1970). Argon laser slit lamp retinal photocoagulation. Transations of the American Academy of Ophthalmology and Otolaryngology, 74, 85-97.

Sand, A. B. (1977). Argon laser treatment of exudative senile maculopathy. Acta Ophthalmologica, 55, 497-506.

Schatz, H., and Patz, A. (1973). Exudative senile maculopathy: I Results of argon laser treatment. Archives of Ophthalmology, 90, 183-196.

Schneider, R. J. (1970). Serous detachment of the macula. Canadian Journal of Ophthalmology, 5, 117-126.

Sorsby, A. (1966). The incidence and causes of blindness in England and Wales 1948-1962. Reports on public health and medical subjects, No. 114. HMSO: London.

Teeters, V. W., and Bird, A. C. (1973a). Neovascularisation in senile disciform macular degeneration. American Journal of Ophthalmology, 75, 53-65.

Teeters, V. W., and Bird, A. C. (1973b). Development of neovascularisation of senile disciform macular degeneration. American Journal of Ophthalmology, 76, 1-18.

Zweng, H. C., Little, H. L., and iPeabody, R. R. (1968). Laser photocoagulation of macular lesions. Transactions of the American Academy of Ophthalmology and Otolaryngology, 72, 377-388. 